\title{
Fatores Associados às Síndromes Hipertensivas em Puérperas Internadas no Hospital Dom Malan em Petrolina-PE: Estudo de Caso-Controle
}

\author{
Lucimara Araújo Campos Alexandre ${ }^{l}$, Gardenia Pereira de Sousa ${ }^{1}$, Lorena Andrade da Silva , \\ Carlos Noronha Neto ${ }^{2}$, Aurélio Antônio Ribeiro da Costa ${ }^{2}$.
}

\begin{abstract}
Resumo: Objetivo: determinar fatores associados às síndromes hipertensivas em puérperas internadas no Hospital Dom Malan (HDM) de Petrolina-PE, nordeste do Brasil. Método: estudo observacional, tipo caso-controle, incluindose puérperas com síndromes hipertensivas (casos) e sem síndromes hipertensivas (controles) na proporção de 1:1, realizado no período de março de 2010 a março de 2012. Como critérios de inclusão para os casos, admitiram-se puérperas com diagnóstico de síndrome hipertensiva na gestação internadas no alojamento conjunto e na Unidade de Terapia Intensiva do HDM e para os controles, puérperas sem síndromes hipertensivas, internadas no alojamento conjunto do HDM. Excluíram-se puérperas com incapacidade cognitiva para entenderem e assinarem o Termo de Consentimento Livre e Esclarecido (TCLE). As variáveis biológicas, sóciodemográficas, hábitos de vida, obstétricas/reprodutivas e principais condições clínicas foram estudadas. Para verificar associação entre variáveis preditoras e o desfecho, utilizaram-se os testes qui-quadrado e exato de Fisher a um nível de significância de 5\%. O intervalo de confiança (IC 95\%) e a Odds Ratio (OR) foram calculados. A análise multivariada foi realizada para determinação do risco ajustado de síndromes hipertensivas na gravidez. Resultados: foram incluídas 150 puérperas (75 casos e 75 controles). Após a análise bivariada os fatores associados às síndromes hipertensivas da gestação foram escolaridade < 9 anos completos de estudo, procedência de Petrolina e história de hipertensão em gestação anterior. Após regressão logística múltipla, apenas a procedência de Petrolina permaneceu como fator de risco para as síndromes hipertensivas da gestação. Conclusão: o estudo demonstrou que mulheres que desenvolveram síndromes hipertensivas na gravidez estavam associadas aos níveis menores de escolaridade, antecedentes de hipertensão em gestações anteriores e serem procedentes de Petrolina. Os achados apresentam algumas limitações, principalmente por se tratar de um estudo observacional. Neste contexto, recomendam-se futuras investigações, como estudos prospectivos maiores em seguimento e número de participantes, sobre potenciais fatores de risco para as síndromes hipertensivas na gravidez na região do Vale do São Francisco.
\end{abstract}

Palavras-chave: Hipertensão Induzida pela Gravidez, Pré-Eclâmpsia, Fatores de Risco, Estudos de Casos e Controles.

\section{Associated factors to Hypertensive syndromes in women interned at the Hospital Dom Malan Petrolina, State of Pernambuco: A Case-Control Study}

\begin{abstract}
Objective: To determine factors associated with hypertensive disorders in women interned at Dom Malan Hospital (HDM) in Petrolina, Pernambuco, in the northeast of Brazil. Method: observational, case-control, including mothers with hypertensive disorders (cases) and without hypertensive disorders (controls) in a 1:1 ratio, conducted from March 2010 to March 2012. As inclusion criteria for cases, mothers diagnosed with arterial hypertension during pregnancy hospitalized in rooming and Intensive Care Unit of the HDM were admitted and for the controls, women with no hypertensive disorders, hospitalized in HDM rooming. We excluded mothers with cognitive disabilities to understand and sign an Informed Consent Form (ICF). The biological, sociodemographic, lifestyle habits, obstetric / reproductive and main clinical conditions were studied. To assess the association between predictor variables and outcome, we used the chi-square and Fisher's exact test to a significance level of 5\%. The confidence interval (95\%) and Odds Ratio (OR) were calculated. Multivariate analysis was performed to determine the adjusted risk of hypertensive disorders in pregnancy. Results: The study included 150 women ( 75 cases and 75 controls). After bivariate analysis, the factors associated with hypertensive disorders of pregnancy were education $<9$ years of study, provenance of Petrolina and history of hypertension in previous pregnancy. After multiple logistic regression, only the provenance of Petrolina remained as a risk factor for hypertensive disorders of pregnancy. Conclusion: The study showed that women who developed hypertensive disorders in pregnancy were associated with lower levels of education, history of hypertension in previous pregnancies and coming from Petrolina. The findings have some limitations, mainly because it is an observational study. In this context, further investigations are recommended, such as larger prospective studies on follow up and numbers of participants, on potential risk factors for hypertensive disorders in pregnancy in the São Francisco Valley.
\end{abstract}

Keywords: Pregnancy-Induced Hypertension, Pre-Eclampsia, Risk Factors, Case-Control Studies.

${ }^{1}$ Universidade Federal do Vale do São Francisco - Pernambuco - Petrolina - Brasil

${ }^{2}$ Instituto de Medicina Integral Prof. Fernando Figueira - Pernambuco - Recife - Brasil. email: lucimara.univasf@gmail.com 


\section{Introdução}

As síndromes hipertensivas na gestação (SHG) merecem especial destaque no cenário da saúde pública mundial, pois configuram a terceira causa de mortalidade materna no mundo e a primeira no Brasil. Em países desenvolvidos, aproximadamente duas a oito, em cada 100 gestantes, desenvolvem o evento, podendo no Brasil chegar a 10\% dos casos. Considera-se ainda importante causa de internamento em unidades de terapia intensiva por apresentarem critérios diagnósticos para morbidade materna grave ${ }^{1}$.

Segundo o National High Blood Pressure Education Program Working Group on High Blood Pressure in Pregnancy $(2000)^{2}$, essas síndromes podem ser classificadas em: hipertensão crônica, pré-eclâmpsia leve, grave e superposta, eclâmpsia e hipertensão gestacional de acordo com a época de surgimento da hipertensão e sua relação com a gravidez, presença de proteinúria e gravidade do quadro. Desses distúrbios, a pré-eclâmpsia e eclâmpsia, se destacam como as principais causas de morbidade e mortalidade materna e perinatal mantendo sua etiologia ainda desconhecida ${ }^{3}$.

Vários são os fatores que estão associados ao aparecimento das síndromes hipertensivas na gestação, dos quais destacamos primiparidade, história familiar de préeclâmpsia, pré-eclâmpsia em gestação anterior (particularmente se for grave, ou antes, da $32^{\mathrm{a}}$ semana de gestação), índice de massa corporal materno pré-gestacional elevado, condições médicas subjacentes que cursam com danos endoteliais (síndrome do anticorpo antifosfolípide, doenças auto-imunes, doença renal crônica, hipertensão crônica, diabetes mellitus pré-gestacional), tabagismo, além dos fatores específicos da gravidez (gestações múltiplas, nuliparidade, mola hidatiforme) ${ }^{4,5,6}$.

Em contrapartida, existem algumas situações em que a associação não está totalmente estabelecida como a idade materna, abortos anteriores e induzidos, intervalo entre gravidezes, primipaternidade, contribuição genética paterna, fatores ambientais (exercício físico, infecções, variação sazonal) e fatores socioeconômicos ${ }^{4,5,6}$.

Desta forma, levando em consideração a ausência de dados locais publicados, nos bancos pesquisados, sobre essa temática para um grupo específico de população proveniente do sertão nordestino, se faz necessário estudar os principais fatores de risco maternos para as síndromes hipertensivas na gravidez em Petrolina e adjacências. Os resultados poderão 
auxiliar na manutenção e/ou aprimoramento de estratégias preventivas para redução de riscos maternos e perinatais.

Portanto, o estudo teve como objetivo de determinar os fatores de risco associados às síndromes hipertensivas em puérperas internadas no Hospital Dom Malan de Petrolina-PE.

\section{Métodos}

O estudo seguiu as orientações da publicação “iniciativa STROBE” para estudos observacionais ${ }^{7}$.

Realizou-se um estudo observacional, analítico, do tipo caso-controle, de base hospitalar que incluiu puérperas com e sem história de hipertensão arterial na gravidez atual, internadas na UTI obstétrica e no alojamento conjunto do Hospital Dom Malan. A coleta dos dados ocorreu no período de abril a julho de 2011 após a aprovação do Comitê de Ética em Pesquisa sob o registro 2215-11. Todas as participantes concordaram voluntariamente em participar e assinaram o termo de consentimento livre e esclarecido.

O cálculo do tamanho da amostra foi realizado no módulo Statcalc do programa EpiInfo 3.5.3, considerando-se uma frequência de pré-eclâmpsia de $6 \%$ em puérperas sem história anterior desse agravo e de 26,4\% em puérperas com esse antecedente (OR=5,58 IC $95 \% 1,81-17,16, \mathrm{p}=0,003)^{5}$, para uma proporção de 1: 1 entre casos e controles. Considerando erro estatístico de 5\%, poder de $80 \%$ e Intervalo de Confiança (IC) de 95\%, chegou-se ao número de 120 participantes. Prevendo-se perdas, acrescentou-se 20\% a esse número, resultando num total de 150 puérperas ( 75 casos e 75 controles).

As síndromes hipertensivas da gravidez foram classificadas segundo os critérios do National High Blood Pressure Education Program (2000) ${ }^{2}$. Como critérios de inclusão para os casos, admitiram-se puérperas com síndrome hipertensiva na gestação internadas no alojamento conjunto e na UTI do HDM, para os controles, puérperas sem síndromes hipertensivas, internadas no alojamento conjunto do HDM que estivessem acomodadas à direita do leito correspondente ao caso. Para cada caso foi admitido um controle. Excluíramse puérperas com incapacidade cognitiva para entenderem e assinarem o Termo de Consentimento Livre e Esclarecido (TCLE). 
As principais variáveis estudadas foram: idade (anos), Índice de Massa Corpórea (IMC) pré-gestacional, raça (branca, negra, parda, amarela e indígena), doenças associadas (diabetes, hipertensão, obesidade), escolaridade (anos completos de estudo), estado civil (união consensual, solteira, casada, separada, viúva), renda per capita (salários mínimos), procedência (Petrolina zona-urbana, Petrolina zona-rural, outras cidades de Pernambuco, outros Estados), tabagismo, etilismo, prática de exercício físico regular antes e durante a gestação, idade da coitarca (anos), número de parceiros, tempo de atividade sexual com o parceiro atual (meses), número de gestações, paridade, história de hipertensão na gravidez em gestação anterior, via de parto (cesárea, vaginal) idade gestacional (IG) no momento do parto (semanas completas), IG da primeira consulta de pré-natal (semanas completas), número de consultas pré-natais, realização de exames, IG do diagnóstico da síndrome hipertensiva (semanas completas), sinais e sintomas de pré-eclâmpsia grave (hipertensão, proteinúria, manifestações visuais e cerebrais e oligúria), uso de sulfato de magnésio, número de internações por alteração da pressão arterial, necessidade de internação em UTI obstétrica.

As mulheres foram entrevistadas no puerpério imediato, internadas nos setores de alojamento conjunto e UTI obstétrica, durante dois turnos semanais. Os prontuários maternos foram consultados. Utilizou-se um formulário padronizado com perguntas fechadas e précodificadas.

A análise estatística foi realizada utilizando o programa estatístico Epi-Info, versão 3.5.3. Na primeira fase descritiva, foram calculadas medidas de tendência central e dispersão para as variáveis quantitativas, obtendo-se também tabelas de distribuição de frequência para as variáveis categóricas. A seguir, uma análise bivariada foi realizada para determinar a associação entre as variáveis independentes (preditoras) e a variável dependente (síndromes hipertensivas). Para tanto, foram utilizados o teste qui-quadrado de associação e o exato de Fisher, quando pertinentes, com nível de significância de 5\%. Calculou-se o Odds Ratio (OR) com intervalo de confiança a 95\%. A análise multivariada foi realizada a partir do modelo teórico causal considerando os níveis hierárquicos das variáveis preditoras em relação a variável desfecho. Realizou-se análise de regressão logística múltipla stepwise, evidenciando para o modelo final, variáveis que persistiram associadas ao desfecho com nível de significância de $5 \%$. 


\section{Resultados}

Durante o período de coleta estiveram internadas na instituição 2400 puérperas. Destas, foram abordadas 150 puérperas, sendo 75 casos e 75 controles, sem recusas nas participações (Figura 1).

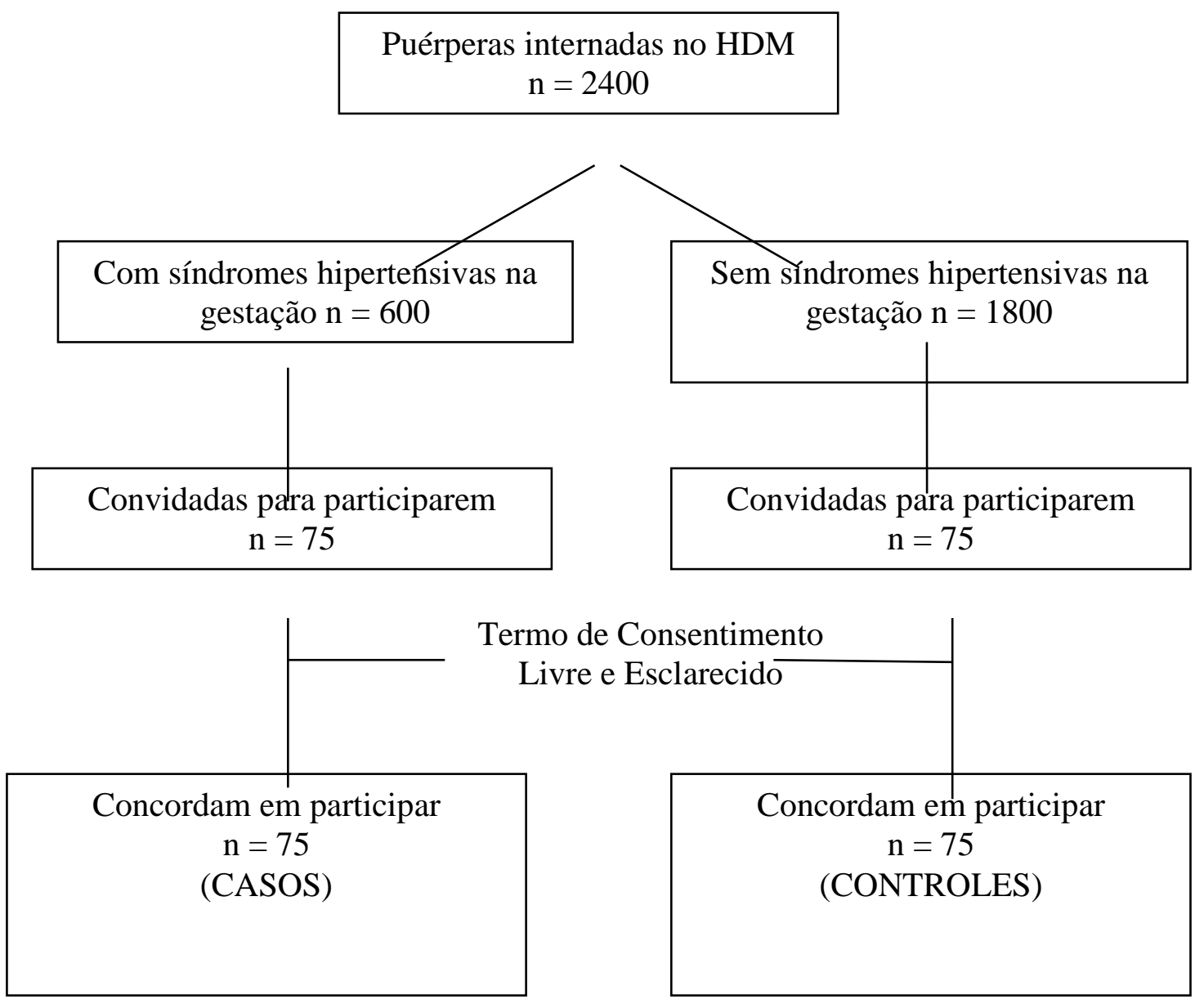

Figura 1: Fluxograma de captação dos participantes

Das 75 puérperas com o diagnóstico de síndrome hipertensiva na gravidez, 58,5\% apresentavam pré-eclâmpsia grave, sendo a forma clínica mais frequente, seguida da pré- 
eclâmpsia leve 14,7\%, hipertensão arterial crônica 8,0\%, hipertensão gestacional 8,0\%, eclâmpsia 6,7\% e pré-eclâmpsia superposta 4,0\% (Tabela 1).

Tabela 1. Distribuição de frequências das síndromes hipertensivas em puérperas internadas no Hospital Dom Malan, Petrolina-PE, 2011.

\begin{tabular}{lcc}
\hline Síndromes Hipertensivas & $\mathrm{n}$ & $\%$ \\
\hline Pré-eclâmpsia grave & 44 & 58,7 \\
Pré-eclâmpsia leve & 11 & 14,7 \\
Hipertensão arterial crônica & 6 & 8,0 \\
Hipertensão gestacional & 6 & 8,0 \\
Eclâmpsia & 5 & 6,7 \\
Pré-eclâmpsia superposta & 3 & 4,0 \\
Total & 75 & 100 \\
\hline
\end{tabular}

n: amostra, \%: porcentagem

A média de idade materna no grupo caso foi de 25,4 \pm 7,36 anos e no grupo controle de 25,2 \pm 6,59 anos, não havendo diferença significativa entre os grupos. A média observada do índice de massa corpórea pré-gestacional no grupo caso foi de $24,2 \pm 4,82 \mathrm{~kg} / \mathrm{m} 2$ e no grupo controle de $23,7 \pm 4,16 \mathrm{~kg} / \mathrm{m} 2$. A raça parda foi a mais prevalente, tanto no grupo caso $(68,0 \%)$ quanto no controle $(65,3 \%)$, seguida da branca e negra. Na presença de doenças préexistentes, nove relataram hipertensão arterial sistêmica prévia, constituindo o grupo das hipertensas crônicas, 5,3\% dos casos e 6,7\% dos controles eram obesas e cinco apresentavam antecedentes de diabetes mellitus, sendo um do grupo caso e quatro do grupo controle respectivamente (Tabela 2 ).

Tabela 2. Distribuição de frequiências das características sociodemográficas das puérperas internadas no Hospital Dom Malan, Petrolina-PE, 2011.

\begin{tabular}{lcc}
\hline & \multicolumn{2}{c}{ Síndromes hipertensivas } \\
\cline { 2 - 3 } Características maternas & Presente & Ausente \\
\hline Idade materna $(\boldsymbol{X} \pm \mathbf{D P})$ & $25,4 \pm 7,36$ & $25,2 \pm 6,59$ \\
IMC pré-gestacional $(\boldsymbol{X} \pm \boldsymbol{D P})$ & $24,2 \pm 4,82$ & $23,7 \pm 4,16$ \\
& & \\
Raça $(\boldsymbol{n} ; \boldsymbol{\%})$ & & \\
Branca & $14 ; 18,7 \%$ & $16 ; 21,3 \%$ \\
Negra & $10 ; 13,3 \%$ & $10 ; 13,3 \%$ \\
Parda & $51 ; 68,0 \%$ & $49 ; 65,3 \%$ \\
\end{tabular}




\section{Doenças associadas (n; \%)}

Diabetes mellitus

Hipertensão arterial sistêmica

Obesidade

Escolaridade (n; \%)

$<9$ anos completos de estudo

$1 ; 1,3 \%$

9; $12 \%$

$4 ; 5,3 \%$

$4 ; 5,3 \%$

$39 ; 52,0 \%$

$36 ; 48,0 \%$

$24 ; 32,0 \%$

$\geq 9$ anos completos de estudo

\section{Estado civil (n; \%)}

Casada

Separada

Solteira

União consensual

Viúva

Renda (n; \%)

$<1$

1

2

3

Procedência(n; \%)

Petrolina zona-rural

Petrolina zona-urbana

Outras cidades de Pernambuco

Outros estados

Tabagismo (n; \%)

$\operatorname{Etilismo}(\boldsymbol{n} ; \%)$

$\begin{array}{cc}24 ; 32 \% & 21 ; 28,0 \% \\ 0 ; 0,0 \% & 1 ; 1,3 \% \\ 8 ; 10,7 \% & 5 ; 6,7 \% \\ 42 ; 56 \% & 48 ; 64,0 \% \\ 1 ; 1,3 \% & 0 ; 0,0 \%\end{array}$

$52 ; 70,3 \%$

$58 ; 79,5 \%$

$16 ; 21,6 \%$

$9 ; 12,3 \%$

$5 ; 6,8 \%$

$2 ; 2,7 \%$

$1 ; 1,4 \%$

4; $5,5 \%$

$\begin{array}{cc}9 ; 12,0 \% & 14 ; 18,7 \% \\ 18 ; 24,0 \% & 40 ; 53,3 \% \\ 16 ; 21,3 \% & 11 ; 14,7 \% \\ 32 ; 42,7 \% & 10 ; 13,3 \% \\ 5 ; 6,7 \% & 1 ; 1,3 \% \\ 3 ; 4,0 \% & 4 ; 5,3 \%\end{array}$

X: média, DP: desvio padrão, n: amostra; \%: percentagem, <: menor, $\geq$ : maior e igual, IMC: índice de massa corpórea.

Do total de registros sobre escolaridade, apenas 48,0\% dos casos possuíam o ensino fundamental completo, enquanto que no grupo controle essa característica foi representada por mais da metade do grupo $(68,0 \%)$. Quanto ao estado civil, tanto no grupo caso como no controle, a união consensual foi a mais prevalente, sendo representada por $56,0 \%$ e $64,0 \%$, respectivamente. No tocante a renda familiar per capita, tanto as puérperas do grupo caso quanto às do grupo controle possuíam rendimento inferior a um salário mínimo em 70,3\% e $79,5 \%$ respectivamente. Em relação à procedência, a maior parte do grupo caso $(42,7 \%)$ era procedente de outros estados, enquanto que no grupo controle, $72,0 \%$ era procedente da cidade de Petrolina (Tabela 2). 
Quanto aos hábitos de vida avaliados, 6,7\% das puérperas do grupo caso e 1,3\% do grupo controle fumavam, 4,0\% dos casos e 5,3\% dos controles ingeriam bebida alcoólica. (Tabela 2).

Referente às características obstétricas e reprodutivas avaliadas nos dois grupos, a média de idade da primeira relação sexual entre os casos foi $17,0 \pm 4,45$ de anos e entre os controles foi de $16,8 \pm 2,71$. Mais da metade dos casos $(56,8 \%)$ possuía um parceiro sexual, enquanto que os controles possuíam dois ou mais parceiros $(51,4 \%)$; a média do tempo de atividade sexual com o parceiro atual foi de $64,1 \pm 66,98$ meses para os casos e de $60,6 \pm 53,4$ para os controles, $41,3 \%$ dos casos e $40,0 \%$ dos controles eram primigestas, $53,3 \%$ dos casos e $46,7 \%$ dos controles eram primíparas e $27,3 \%$ dos casos e $11,1 \%$ dos controles relataram hipertensão em gravidez anterior (Tabela 3).

Tabela 3. Distribuição de frequiências das características clínico-obstétricas das puérperas internadas no Hospital Dom Malan, Petrolina-PE, 2011.

\begin{tabular}{lcc}
\hline \multirow{2}{*}{ Características maternas } & \multicolumn{2}{c}{ Síndromes hipertensivas } \\
& Presente & Ausente \\
\hline Idade da coitarca $(\boldsymbol{X} \pm \boldsymbol{D P})$ & $17,0 \pm 4,45$ & $16,8 \pm 2,71$
\end{tabular}

Número de parceiros (n; \%)

Um parceiro

$42 ; 56,8 \%$

$32 ; 43,2 \%$

$64,1 \pm 66,98$

$(X \pm D P)$

Número de gestações (n; \%)

Primigestas

Não-primigestas

Paridade (n; \%)

Primíparas

Multíparas

Hipertensão em gravidez anterior (n; \%)

$I G$ da primeira consulta de pré-natal $(X \pm D P)$

Número de consultas pré-natais (n; \%)

$<6$ consultas pré-natais

$\geq 6$ consultas pré-natais
$31 ; 41,3 \%$

$44 ; 58,7 \%$

$30 ; 40,0 \%$

$45 ; 60,0 \%$

$40 ; 53,3 \%$

$35 ; 46,7 \%$

$35 ; 46,7 \%$

$12 ; 27,3 \%$

$40 ; 53,3 \%$

$13,0 \pm 5,99$

$5 ; 11,1 \%$

$12,9 \pm 5,42$

$25 ; 33,3 \%$

$50 ; 66,6 \%$

$18 ; 24,0 \%$

$57 ; 76,0 \%$ 


\begin{tabular}{|c|c|c|}
\hline Realização de exames de rotina do pré-natal (n; \%) & $73 ; 97,3 \%$ & $75 ; 100,0 \%$ \\
\hline IG do dianóstico da síndrome hipertensiva $(X \pm D P)$ & $32,2 \pm 7,52$ & - \\
\hline \multicolumn{3}{|l|}{ Sinais e sintomas de pré-eclâmpsia grave (n; \%) } \\
\hline $\mathrm{PAS} \geq 160 \mathrm{mmHg}$ e/ou PAD $\geq 110 \mathrm{mmHg}$ & $47 ; 62,7 \%$ & - \\
\hline Proteinúria $\geq 2 \mathrm{~g} / 24 \mathrm{~h}$ ou $3+$ na fita & $41 ; 54,7 \%$ & - \\
\hline Manifestações visuais & $21 ; 28,0 \%$ & - \\
\hline Manifestações cerebrais & $43 ; 57,3 \%$ & - \\
\hline Oligúria & $2 ; 2,7 \%$ & - \\
\hline Edema & $48 ; 64,0 \%$ & - \\
\hline Internação por alteração da pressão arterial (n; \%) & $15 ; 20,5 \%$ & $0 ; 0,0 \%$ \\
\hline Número de internações (mediana; variação) & $1 ; 1-4$ & 0 \\
\hline IG da terminação do parto $(X \pm D P)$ & $36,5 \pm 3,19$ & $38,4 \pm 2,61$ \\
\hline \multicolumn{3}{|l|}{ Tipo de parto (n; \%) } \\
\hline Cesariana & $59 ; 78,7 \%$ & $36 ; 48,0 \%$ \\
\hline Vaginal & $16 ; 21,3 \%$ & $39 ; 52,0 \%$ \\
\hline Internação em UTI obstétrica (n; \%) & $56 ; 74,7 \%$ & $2 ; 2,7 \%$ \\
\hline Uso do sulfato de magnésio (n; \%) & $56 ; 74,7 \%$ & - \\
\hline
\end{tabular}

X: média, DP: desvio padrão, n: amostra; \%: percentagem, <: menor, $\geq:$ maior e igual, g: gramas, h: horas, IG: idade gestacional, PAS: pressão arterial sistólica, PAD: pressão arterial diastólica, mmHg: milímetros de mercúrio.

Quando analisadas quanto às condições clínicas do pré-natal, a média de idade gestacional na primeira consulta foi de 13,0 $\pm 5,99$ semanas para os casos e de 12,9 $\pm 5,42$ para os controles; a maior parte das puérperas, tanto do grupo caso $(66,6 \%)$ quanto do grupo controle $(76,0 \%)$, realizaram consultas pré-natais numa frequência maior ou igual a seis consultas e $97,3 \%$ dos casos e 100,0\% dos controles realizaram exames de rotina do pré-natal. A hipertensão na gravidez no grupo caso foi diagnosticada, em média, a partir da $32^{\mathrm{a}}$ semana de gestação (Tabela 3).

Quando as puérperas, com diagnóstico de síndromes hipertensivas (grupo caso), foram avaliadas quanto à presença de sinais e sintomas de pré-eclâmpsia grave, encontrou-se 62,7\% com PAS $\geq 160 \mathrm{mmHg}$ e/ou PAD $\geq 110 \mathrm{mmHg}, 54,7 \%$ proteinúria $\geq 2 \mathrm{~g} / 24 \mathrm{~h}$ ou $3+$ na fita, 28,0\% manifestações visuais, 57,3\% manifestações cerebrais, 2,7\% oligúria e 64,0\% edema. Quanto à internação por alteração da pressão arterial, avaliada no mesmo grupo, 20,5\% relatou ter sido internada por alteração da pressão arterial durante o período gravídico, variando o número de internações de uma a quatro vezes (Tabela 3). 
No momento do parto, a média da idade gestacional no grupo caso foi de $36,5 \pm 3,19$ semanas e no grupo controle foi de $38,4 \pm 2,61$. O parto vaginal foi assistido em $21,3 \%$ das mulheres no grupo caso e em 52,0\% no grupo controle, enquanto que à interrupção por cesariana se deu em 78,7\% do grupo caso e em 48,0\% no grupo controle. Foram encaminhadas a UTI obstétrica e fizeram uso do sulfato de magnésio no pós-parto $74,7 \%$ do grupo das hipertensas (Tabela 3).

Analisando a associação entre os fatores de risco maternos, observou-se que puérperas com síndromes hipertensivas na gestação, tinham maior chance de apresentarem escolaridade menor que nove anos completos de estudo ( $\mathrm{OR}=0,43$; IC 95\%; 0,22 - 0,84), serem procedentes de Petrolina (OR=0,22; IC 95\%; 0,11 - 0,44) e terem história de hipertensão em gravidez anterior $(\mathrm{OR}=3,00$; IC 95\%; 0,98 - 9,40). Não houve associação entre síndrome hipertensiva na gravidez e idade materna $<20$ anos $(\mathrm{p}=0,15)$, idade materna $>40$ anos $(\mathrm{p}=0,18)$, IMC pré-gestacional $>30 \mathrm{~kg} / \mathrm{m}^{2}(\mathrm{p}=0,98)$, sem companheiro $(\mathrm{p}=0,41)$, renda per capta $<$ um salário mínimo $(\mathrm{p}=0,26)$, tabagismo $(\mathrm{p}=0,10)$, etilismo $(\mathrm{p}=0,50)$, idade da coitarca $\leq 17$ anos $(\mathrm{p}=0,86)$, número de parceiros $>$ um $(\mathrm{p}=0,32)$, tempo de atividade sexual com o parceiro atual $(\mathrm{p}=0,86)$, primigesta $(\mathrm{p}=0,87)$ e primiparidade $(\mathrm{p}=0,41)$ (Tabela 4$)$.

Tabela 4. Associação entre as variáveis independentes e o aparecimento de síndrome hipertensiva na gravidez em puérperas internadas no Hospital Dom Malan, Petrolina-PE, 2011.

\begin{tabular}{|c|c|c|c|c|c|c|c|}
\hline \multirow{3}{*}{ Fatores associados } & \multicolumn{4}{|c|}{ Síndromes hipertensivas } & \multirow{3}{*}{ OR } & \multirow{3}{*}{ IC $95 \%$} & \multirow{3}{*}{$p$} \\
\hline & \multicolumn{2}{|c|}{$\begin{array}{c}\text { SIM } \\
(\mathrm{n}=75)\end{array}$} & \multicolumn{2}{|c|}{$\begin{array}{c}\mathrm{NÃO} \\
(\mathrm{n}=75)\end{array}$} & & & \\
\hline & $\mathrm{n}$ & $\%$ & $\mathrm{n}$ & $\%$ & & & \\
\hline Idade materna $\leq 20$ anos & 25 & 33,3 & 17 & 22,7 & 0,59 & $0,28-1,21$ & $0,15^{*}$ \\
\hline Idade materna $\leq 40$ anos & 4 & 5,3 & 1 & 1,3 & 4,17 & $0,46-38,21$ & $0,18^{* *}$ \\
\hline $\begin{array}{l}\text { IMC pré-gestacional > } 30 \\
\mathrm{~kg} / \mathrm{m}^{2}\end{array}$ & 8 & 10,6 & 8 & 10,6 & 1,02 & $0,36-2,87$ & $0,98 *$ \\
\hline Raça negra & 10 & 13,3 & 10 & 13,3 & 1,00 & $0,39-2,56$ & $1,00 *$ \\
\hline Escolaridade $<9$ anos & 39 & 52,0 & 24 & 32,0 & 0,43 & $0,22-0,84$ & $0,01^{*}$ \\
\hline Sem companheiro & 9 & 12,0 & 6 & 8,0 & 0,64 & $0,22-1,89$ & $0,41 *$ \\
\hline $\begin{array}{l}\text { Renda per capta }<1 \text { salário } \\
\text { mínimo }\end{array}$ & 52 & 69,3 & 58 & 77,3 & 0,66 & $0,32-1,36$ & $0,26 *$ \\
\hline Procedência de Petrolina & 27 & 36,0 & 54 & 72,0 & 0,22 & $0,11-0,44$ & $<0,01 *$ \\
\hline Tabagismo & 5 & 6,7 & 1 & 1,3 & 5,28 & $0,60-46,38$ & $0,10^{* *}$ \\
\hline Etilismo & 3 & 4,0 & 4 & 5,3 & 0,74 & $0,16-3,42$ & $0,50 * *$ \\
\hline
\end{tabular}


Id on Line Revista Multidisciplinar e de Psicoloqia

Id on Line Multidisciplinary and Psycology Journal

Número de parceiros $>1$

Tempo de atividade sexual

com o parceiro atual

Primigesta

Primiparidade

Hipertensão em gravidez

anterior

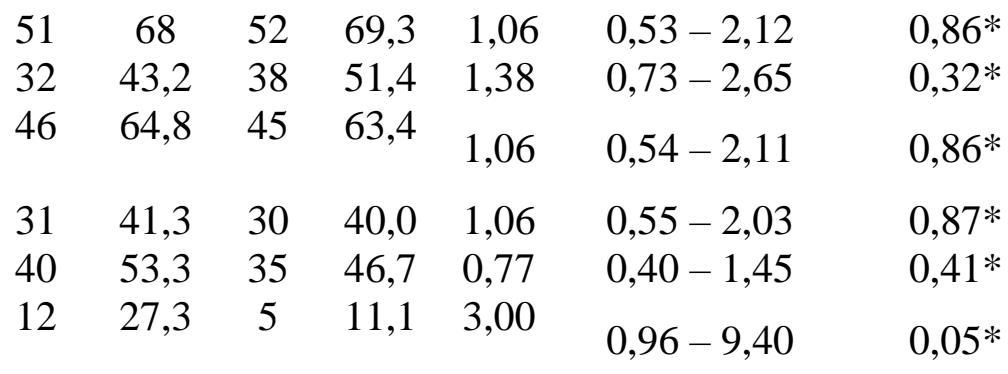

n: amostra, \%: porcentagem, OR: odds ratio, IC: Intervalo de Confiança a 95\%, $P$ : significância, IMC: Indice de Massa Corporal, $\leq$ : menor e igual, $\geq$ : maior e igual, $>$ :maior, $<:$ menor, $\mathrm{kg} / \mathrm{m}^{2}$ : quilograma por metro quadrado.

* Teste Chiquadrado, ** Teste Exato de Fisher.

Após análise multivariada, através de regressão logística por modelo hierárquico, apenas procedência de Petrolina (OR=0,22; IC 95\%; 0,11 - 0,44) persistiu significativamente associada com o risco aumentado de síndromes hipertensivas (Tabela 5).

Tabela 5. Modelo final de regressão logística

\begin{tabular}{lrrrrrrrr}
\hline \hline Term & $\begin{array}{l}\text { Odds } \\
\text { Ratio }\end{array}$ & $\mathbf{9 5 \%}$ & C.I. & Coefficient & S. E. & $\begin{array}{c}\text { Z- } \\
\text { Statistic }\end{array}$ & $\begin{array}{c}\text { P- } \\
\text { Value }\end{array}$ \\
\hline $\begin{array}{l}\text { PROCEDENCIA1 } \\
\text { (Yes/No) }\end{array}$ & $\underline{0,2188}$ & $\underline{0,1097}$ & $\underline{0,4362}$ & $-1,5198$ & 0,3521 & $-4,3161$ & $\underline{0,0000}$ \\
CONSTANT & $*$ & $*$ & $*$ & 0,8267 & 0,2616 & 3,1599 & $\underline{0,0016}$ \\
\hline
\end{tabular}

C.I.: intervalo de confiança, S.E.: erro padrão.

Fonte: Epi-Info versão 3.5.1

\section{Discussão}

Embora reconhecida desde a antiguidade, a compreensão das causas da pré-eclâmpsia é ainda limitada. Fatores maternos, pré-concepcionais e associados à gravidez têm sido descritos. Apesar de uma extensa pesquisa, essas observações ainda permanecem como principais fatores de risco e preditores dos distúrbios hipertensivos associados à gestação.

Consistente com a idéia de que a pré-eclâmpsia pode ser materna (início atrasado), placentária (início precoce) ou uma associação de fatores em sua origem, fatores de risco 
conhecidos para as síndromes hipertensivas têm sido associados com a gravidez e podem ter diferentes efeitos na primeira gestação quando comparada às subsequentes. Fatores de risco paternos e ambientais também têm sido sugeridos.

O presente estudo evidenciou que, entre as puérperas com síndromes hipertensivas da gestação, a forma clínica mais frequente foi a pré-eclâmpsia grave $(n=40 ; 57,1 \%)$. Resultados semelhantes em frequência foram observados em outro estudo ${ }^{4}$ também realizado em hospital de nível terciário, que avaliou 201 pacientes com síndromes hipertensivas, encontrando uma prevalência de $52,7 \%$ de pré-eclâmpsia grave. Já outros relatos, com metodologia semelhante, destacaram valores inferiores ${ }^{5,8,9}$.

Os extremos de idade materna $(\leq 20$ e $\geq 40$ anos $)$ embora comprovadamente responsáveis pelo aumento do risco de pré-eclâmpsia e hipertensão crônica ${ }^{10,11,12}$, não apresentaram significância estatística quando associados às síndromes hipertensivas em nosso estudo, $\mathrm{p}=0,15$ e $\mathrm{p}=0,18$, respectivamente. Acredita-se que esse dado possa ser modificado à medida que aumente o número de participantes da amostra. Em revisão sistemática ${ }^{4}$ incluindo 52 estudos (coorte e caso-controle), mulheres com mais de 40 anos tinham quase o dobro do risco de desenvolver pré-eclâmpsia quando comparadas às mulheres mais jovens. O aumento do risco foi semelhante em primíparas e multíparas. Quanto à associação entre idade materna jovem e o risco para síndromes hipertensivas, parece não existir consenso na literatura ${ }^{4,10,12}$.

A possível explicação para a ausência de diferenças significativas quanto à idade e o risco de hipertensão na gravidez em nossos resultados, talvez se deva pelo tamanho amostral ter sido desenhado para outra variável e esta diferença pode não ter aparecido, correspondendo ao erro estatístico tipo II, onde as possíveis diferenças não apareçam pelo número reduzido de participantes. Esta situação, certamente pode ser destacada como uma limitação metodológica, característica do desenho de estudo em questão.

Considerando o IMC pré-gestacional $\geq 30 \mathrm{~kg} / \mathrm{m}^{2}$, o estudo não evidenciou associação com o surgimento de hipertensão na gravidez. Sabe-se, no entanto, que um elevado índice de massa corporal pré-gestacional tem sido constantemente encontrado aumentando o risco de pré-eclâmpsia, com odds ratio relatado entre três e cinco para obesas (IMC>30) em comparação com mulheres de peso adequado ${ }^{4}$. Este achado é consistente com a idéia de que um IMC elevado esteja associado a aumento da resposta inflamatória vascular levando a gravidez a um estado de má adaptação placentária e consequente pré-eclâmpsia ${ }^{4,11,13,14}$. Como 
a média do IMC ficou muito semelhante entre os grupos (cerca de $24,0 \mathrm{~kg} / \mathrm{m}^{2}$ ) esta homogeneidade pode ter influenciado no não aparecimento da diferença. Além disso, as mulheres da região que contribuem para clientela do hospital em questão possuem características nutricionais parecidas, o que poderia ocultar a diferença para este número de participantes.

No que diz respeito à raça das mulheres, também não constituiu, neste estudo, fator de risco para síndromes hipertensivas. Porém, estudos evidenciaram que mulheres negras têm um risco duas vezes maior de desenvolverem pré-eclâmpsia quando comparadas às mulheres brancas. Há também uma probabilidade maior do primeiro grupo ter hipertensão crônica fora do status gravídico-puerperal ${ }^{4,15}$. Esta condição talvez seja explicada pela diversidade racial com que é constituída a nação brasileira em geral e a nossa população, em particular. É sabido que o estudo de fatores associados à raça em nossa realidade fica revestido por alguns cuidados devido a nossa sociedade ser construída por uma grande miscigenação, sem possuirmos estratos raciais bem definidos. No entanto, é sabido que as mulheres de cor negra são mais propensas a desenvolver hipertensão, e isto por si, pode ser fator relevante para o aparecimento de pré-eclâmpsia nestas pacientes.

Resultados semelhantes foram relatados por Dempsey e colaboradores $(2003)^{16}$, que também relataram risco aumentado entre mulheres africano-americanas quando comparadas às brancas ou hispânicas e por Knuist e colaboradores (1998) ${ }^{17}$ que encontraram risco dobrado em mulheres negras quando comparadas com às brancas. Mulheres de outras origens étnicas (Mediterrâneo, Ásia ou outros) também tiveram maiores riscos, mas os números eram pequenos e as estimativas não alcançaram significância.

Avaliando as condições médicas subjacentes (diabetes mellitus, hipertensão arterial sistêmica e obesidade) como possíveis fatores de risco para hipertensão na gravidez, nenhum dos agravos se mostrou associado ao desfecho. Estudo observacional mostrou que précondições médicas subjacentes associadas aos danos endoteliais como diabetes mellitus, síndrome dos anticorpos antifosfolípedes, doenças auto-imunes e renais, estavam fortemente associadas com um risco aumentado de pré-eclâmpsia ${ }^{12}$. Para o diabetes mellitus, as taxas de incidência variavam entre $9 \%$ e $66 \%$ em mulheres com nefropatia diabética pré-existente ${ }^{18}$. Sabe-se também que a hipertensão crônica é um importante fator de risco para o desenvolvimento de pré-eclâmpsia superposta ${ }^{19}$. Talvez por se tratar de um hospital terciário, 
as pacientes do estudo, tanto as com doenças pregressas como as que não as apresentam, procuram o estabelecimento para uma melhor assistência o que poderia modificar o aparecimento desta associação.

A associação entre pré-eclâmpsia e história familiar é controversa em diversos estudos ${ }^{11,20}$. Uma revisão sistemática inglesa, que reuniu mais de cinquenta estudos casocontroles e coortes, com 23 deles envolvendo mais de 100 mulheres, evidenciou que a presença de mãe ou irmã com pré-eclâmpsia triplicaria o risco de desenvolver este agravo e que mulheres com pré-eclâmpsia grave, provavelmente possuíam mães que também apresentaram o quadro clínico em questão ${ }^{11}$. De forma contrária, estudo de base hospitalar não confirmou a história familiar como fator de risco para pré-eclâmpsia ${ }^{20}$.

Considerando os fatores socioeconômicos avaliados nesse estudo (escolaridade, renda e procedência), escolaridade $(\mathrm{p}=0,01)$ e procedência $(\mathrm{p}<0,01)$ se mostraram associadas ao desfecho. Apesar de o baixo nível socioeconômico ser um importante fator de risco para a pré-eclâmpsia, de acordo com o trabalho de Silva e colaboradores $(2008)^{21}$, parece que esta associação está mais relacionada à escolaridade (OR 5,12, IC 95\% 2,20-11,93 para baixa escolaridade), caracterizando-se como uma variável de confundimento. Os autores não observaram associação entre nível socioeconômico e pré-eclâmpsia ${ }^{22}$. O nosso estudo demonstrou resultados semelhantes ao deste trabalho e apesar dos fatores socioeconômicos terem sido propostos influenciando o risco de pré-eclâmpsia, os dados permanecem inconclusivos e conflitantes ${ }^{4}$.

O estudo não demonstrou aumento no risco entre fumar e desenvolver hipertensão na gravidez. O uso do cigarro na gestação também não foi associado com o aumento do risco para pré-eclâmpsia em outro estudo semelhante que tinha como objetivo determinar esta $\operatorname{associação~}^{23}$

Destacada em alguns estudos como risco para a pré-eclâmpsia, mas não para hipertensão crônica ${ }^{10,24,25}$, a primiparidade não constituiu fator de risco nessa amostra estudada. Este resultado não corroborou com outro estudo realizado na Noruega, no qual a prevalência de pré-eclâmpsia em mulheres primíparas de acordo com o Medical Birth Registry of Norway da Noruega (MBRN) foi de 5,1\% ${ }^{26}$. Em recente meta-análise sobre os efeitos e mecanismos da primiparidade e o risco de pré-eclâmpsia, 26 estudos evidenciaram uma razão de chance de duas vezes para pré-eclâmpsia em primigestas em comparação a 
mulheres multíparas (OR 2,42; IC 95\% 2,16-2,71) ${ }^{26}$. O aumento do risco persistiu após o ajuste para fatores de confusão. Odegard e colaboradores (2000) relataram estimativas semelhantes em um estudo de caso-controle bem conduzido, onde participaram 973 mulheres sendo 323 casos e 650 controles $^{27}$.

Acredita-se que a nuliparidade tem maior expressão como fator de risco para as síndromes hipertensivas em países desenvolvidos onde o perfil da primigesta tem se modificado com aumento do número de mulheres programando a gestação para depois dos 30 anos. Estima-se um risco de recorrência maior que $40 \%$ para nulíparas acometidas com préeclâmpsia após a trigésima semana de gestação $^{24}$. Talvez nossa população possua características peculiares como início diferenciado da idade em que começam a engravidar. Como são mulheres mais jovens, de região mais pobre, esta associação não tenha aparecido, principalmente pelo número reduzido de participantes com cronologia suficiente para apresentarem este antecedente.

No que se refere à história de hipertensão em gravidez anterior, os artigos publicados na literatura revelam esta situação como fator de risco para gravidezes subsequentes, com risco de recorrência de $14 \%$, principalmente em mulheres multíparas ${ }^{4}$. Em concordância, nosso estudo demonstrou associação desta variável com o desenvolvimento de hipertensão na gravidez. Em revisão sistemática dos fatores de risco para a pré-eclâmpsia, Duckitt e Harrington $(2005)^{11}$ relataram aumento de sete vezes no risco em mulheres com préeclâmpsia prévia em comparação com as mulheres sem precedentes de pré-eclâmpsia. Mulheres com pré-eclâmpsia recorrente são mais propensas a desenvolverem pré-eclâmpsia grave nas próximas gravidezes, maior risco de parto prematuro, descolamento prematuro de placenta e morte fetal ${ }^{11}$.

Gestantes que apresentem hipertensão na gravidez deveriam ser aconselhadas quanto ao risco de desenvolverem hipertensão em gravidezes futuras, visto que há relatos de recorrência associada ao número de gestações subsequentes, onde esse risco pode ser aumentado em até sete vezes ${ }^{11}$. Em contrapartida, poucas mulheres desenvolvem formas graves de hipertensão na segunda gestação respeitando os casos de trombofilias, colagenoses e síndrome do anticorpo antifosfolípide, onde essa afirmativa não é verdadeira ${ }^{20,28}$.

Considerando os resultados do presente estudo apenas a variável procedência $(p<0,01)$ manteve uma associação significativa após a realização do modelo final de regressão 
logística. Este achado peculiar pode fomentar algumas conjecturas como, por exemplo, a característica das mulheres que residem mais próximas ao hospital. Estas mulheres apresentariam nível de entendimento melhor com relação aos cuidados na saúde do que as de localidades mais distantes ou mesmo zonas rurais mais isoladas. Além disso, deve-se sempre levar em consideração que, por ser um hospital terciário, as pessoas que procuram o estabelecimento esperam por uma melhor assistência, o que pode distorcer possíveis associações. Da mesma forma, as mulheres que apresentarem riscos mais elevados para complicações procurarão o hospital mais precocemente e isto poderia influenciar positivamente, com um controle mais rigoroso e adequado da hipertensão ou outras doenças associadas. Por fim, mesmo usando um tratamento metodológico que exclui algumas variáveis de confusão, estas ainda poderiam influenciar o modelo causal e se apresentar como uma situação de proteção o que pode ser observado em estudos observacionais.

Ainda com relação à característica do tipo de estudo, algumas limitações podem sempre ser destacadas, o número reduzido de participantes, pode não evidenciar algumas diferenças que, por ventura, existiriam, o que denota obstáculo metodológico. Além disso, deve-se considerar a particularidade das pessoas da região, principalmente por se tratar de uma população com características próprias e podendo estas desempenhar algum papel na influência da elevação dos riscos para se apresentar pré-eclâmpsia na gestação. Identificar estes fatores, em outros estudos com um número maior de participantes, levando em consideração as variáveis menos frequentes, é situação determinante para construir um perfil da gestante de risco para as síndromes hipertensivas para cada região específica, considerando além dos riscos já estabelecidos na comunidade científica, e assim, transpor estes resultados para a comunidade.

Recomendam-se, ainda, futuras investigações que deverão contemplar outras variáveis e a realização de estudos prospectivos sobre potenciais fatores de risco para as síndromes hipertensivas na gravidez.

Para fins práticos, as mulheres em maior risco podem ser identificadas e devem ter seguimento rigoroso. Nenhuma ação preventiva primária específica está disponível. Prevenção das graves consequências de pré-eclâmpsia ainda depende da detecção precoce do aumento da pressão arterial e proteinúria. A assistência pré-natal tem importante papel nessa prevenção, uma vez que se observa que mulheres desprovidas de acompanhamento pré-natal 
são sete vezes mais propensas ao óbito decorrente de complicações relacionadas à préeclâmpsia e eclâmpsia quando comparadas às mulheres que receberam o mínimo de assistência segundo a Organização Mundial de Saúde (OMS) ${ }^{24}$.

\section{Referências}

1. Noronha NC, Souza ASR de, Amorim MMR. Tratamento da pré-eclâmpsia baseado em evidências. Rev Bras Ginecol Obstet 2010; 32: 459-68.

2. National High Blood Pressure Education Program: Report of the National High Blood Pressure Education Program Working Group on high blood pressure in pregnancy. Am J Obstet Gynecol. 2000; 183: S1-S22.

3. Souza ASR, Amorim MR, Costa AAR, Noronha Neto C. Tratamento anti-hipertensivo na gravidez. Acta Med Port. 2010; 23: 077-84.

4. Trogstad L, Magnus P, Stoltenberg C. Pre-Eclampsia: Risk factors and causal models. Best Pract Res Clin Obstet Gynaecol. 2011; 25: 329-42.

5. Assis TR, Viana FP, Rassi S. Estudo dos principais fatores de risco maternos nas síndromes hipertensivas da gestação. Arq. Bras. Cardiol. 2008; 91: 11-17.

6. Gaio DS, Schmidt MI, Duncan BB, Nucci LB, Matos MC, Branchtein L. Hypertensive disorders in pregnancy: frequency and associated factors in a cohort of brazilian women. Hypertens Pregnancy. 2011; 20: 269-81.

7. Malta M, Cardoso LO, Bastos FI, Ferreira MM, Passos MCF. STROBE initiative: guidelines on reporting observational studies. Rev Saúde Pública 2010; 44: 559-65.

8. Costa HLFF, Costa CFF, Costa LOBF. Idade materna como fator de risco para a hipertensão induzida pela gravidez: análise multivariada. Rev Bras Ginecol Obstet. 2003; 25: 631-5.

9. Cabral SALCS, Costa CFF, Cabral Jr SF. Correlação entre a idade materna, paridade, gemelaridade, síndrome hipertensiva e ruptura prematura de membranas e a indicação de parto cesáreo. Rev Bras Ginecol Obstet. 2003; 25: 739-44.

10. Robillard PY, Dekker G, Chaouat G, Hulsey TC. Etiology of preeclampsia: maternal vascular predisposition and couple disease-mutual exclusion or complementarity? J Reprod Immunol. 2007; 76: 1-7. 
11. Duckitt K, Harrington D. Risk factors for pre-eclampsia at antenatal booking: systematic review of controlled studies. BMJ. 2005; 330: 565.

12. Tanaka M, Jaamaa G, Kaiser M, Hills E, Soim A, Zhu M et al. Racial disparity in hypertensive disorders of pregnancy in New York State: a 10-year longitudinal population-based study. Am J Public Health. 2007; 97: 163-70.

13. Samuels-Kalow ME, Funai EF, Buhimschi C, Norwitz E, Perrin M, Calderon-Margalit $\mathrm{R}$ et al. Pregnancy body mass index, hypertensive disorders of pregnancy, and long term maternal mortality. Am J Obstet Gynecol 2007; 197: 490.

14. Chandiramani M, Shennan A. Hypertensive disorders of pregnancy: a UK-based perspective. Curr. Opin. Obstet. Gynecol. 2008; 20: 96-101.

15. Sibai BM, Ewell M, Levine RJ, Klebanoff MA, Esterlitz J, Catalano PM et al. Risk factors associated with preeclampsia in healthy nulliparous women. Am J Obstet Gynecol 1997; 177: 1003-10.

16. Dempsey JC, Williams MA, Luthy DA, Emanuel I, Shy K. Weight at birth and subsequent risk of preeclampsia as an adult. Am J Obstet Gynecol 2003; 189: 494500.

17. Knuist M, Bonsel GJ, Zondervan HA, Treffers PE. Risk factors for preeclampsia in nulliparous women in distinct ethnic groups: a prospective cohort study. Obstet Gynecol 1998; 92: 174-8.

18. Sibai BM. Risk factors, pregnancy complications, and prevention of hypertensive disorders in women with pregravid diabetes mellitus. J Matern Fetal Med 2000; 9: 625 .

19. Chappell LC, Enye S, Seed P, Briley AL, Poston L, Shennan AH. Adverse perinatal outcomes and risk factors for preeclampsia in women with chronic hypertension: a prospective study. Hypertension 2008; 51: 1002-9.

20. Livingston JC, Maxwell BD, Sibai BM. Chronic hypertension in pregnancy. Minerva Ginecol. 2003; 55: 1-13.

21. Silva LM, Coolman M, Steegers EAP, Jaddoe VW, Moll HA, Hofman A et al. Low socioeconomic status is a risk factor for preeclampsia: the Generation R Study. J Hypertens 2008; 26: 1200-08.

22. Lawlor DA, Morton SMB, Nitsch D et al. Association between childhood and adulthood socioeconomic position and pregnancy induced hypertension: results from the Aberdeen children of the 1950s cohort study. J Epidemiol Comm Health 2005; 59: 49-55. 
23. England L, Zhang J. Smoking and risk of preeclampsia: a systematic review. Front Bioscience 2007; 12: 2471-83.

24. Wagner LK. Diagnosis and management of preeclampsia. Am Fam Physician. 2004; 70: 2317-24.

25. Villar J, Carroli G, Wojdyla D, Abalos E, Giordano D, Ba'ageel H et al. Preeclampsia, gestacional hypertension and intrauterine growth restriction, related or independent conditions? Am J Obstet Gynecol. 2006; 194: 921-31.

26. Luo ZC, An N, Xu HR, Larante A, Audibert F, Fraser WD. The effects and mechanisms of primiparity on the risk of pre-eclampsia: a systematic review. Paediatr Perinat Epidemiol 2007; 21(1): 36-45.

27. Ødegård R, Vatten LJ, Nilsen ST et al. Risk factors and clinical manifestations of preeclampsia. BJOG 2000; 107: 1410-6.

28. Hiartardottir S, Leifsson BG, Geirsson RT, Steinthorsdottir V. Recurrence of hypertensive disorder in second pregnancy. Am J Obstet Gynecol 2006; 194: 916-20.

\section{Como citar este artigo (Formato ABNT):}

ALEXANDRE Lucimara A.C.; SOUSA, Gardenia P. de; SILVA, Lorena A. da; NORONHA NETO, Carlos; COSTA, Aurélio A.R da. Fatores Associados às Síndromes Hipertensivas em Puérperas Internadas no Hospital Dom Malan em Petrolina-PE: Estudo de Caso-Controle. Id on Line Revista Multidisciplinar e de Psicologia, 2017, vol.11, n.37, p. 329-347. ISSN: 1981-1179.

Recebido: 26.08 .2017

Aceito: 29.08 .2017 